\title{
Planned Staged Repair of Thoracoabdominal Aortic Aneurysms to Minimize Spinal Cord Injury A Proof of Concept
}

\author{
Gabriele Di Luozzo, MD*, Michael Wilderman, MD', Amit Pawale, MD², \\ Jock McCullough, MD', Randall B. Griepp, MD² \\ ${ }^{1}$ Heart and Vascular Hospital, Hackensack University Medical Center, Hackensack, New Jersey, USA \\ ${ }^{2}$ Department of Cardiovascular Surgery, Icahn School of Medicine at Mount Sinai, New York, New York, USA
}

\begin{abstract}
Management of thoracoabdominal aortic aneurysms (TAA) can lead to spinal cord injury. A variety of clinical adjuncts have proven to decrease the incidence of paraplegia; however, at least $10 \%$ patients remain at risk of developing paraplegia. Experimentally and in sporadic clinical experiences, the staged repair of TAAs can lead to better neurologic outcomes. We present two clinical cases with extensive TAA in which a deliberate staged repair leads to excellent neurologic outcomes.

Copyright (c) 2015 Science International Corp.
\end{abstract}

\section{Key Words}

Thoracoabdominal aortic aneurysm - Paraplegia • Spinal cord

\section{Introduction}

The repair of thoracoabdominal aortic aneurysms (TAAs) remains a formidable challenge for most cardiovascular surgeons. One of the main goals in the management of patients with an extensive thoracic aortic aneurysm is end organ protection, both viscera and spinal cord. Early postoperative morbidity and mortality remains high when permanent spinal cord injury $(\mathrm{SCl})$ occurs. The incidence of paraplegia increases when the segmental arteries (SAs) that are

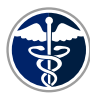

Fax +1 2037853552

E-Mail: aorta@scienceinternational.org

http://aorta.scienceinternational.org (c) 2015 AORTA

Published by Science International Corp. ISSN 2325-4637

Accessible online at:

http://aorta.scienceinternational.org sacrificed start from the lower thorax and extend into the abdomen and when the number sacrificed is between 8 to 12 [1]. However, when the distal aorta is approached in two or more stages the incidence of spinal cord injury decreases significantly [2]. Experimental and clinical data suggests that staged SA sacrifice leads to a less dramatic drop in collateral network perfusion pressure (CNPP) during the second stage, an increase in arterial diameter and density of the intraspinous and paraspinous vessels, and a greater margin of safety to prevent $\mathrm{SCl}$.

The patient's cardiopulmonary status and anatomical features of the TAA may not afford a staged repair due to significant thrombus burden, calcification, and uniform dilatation throughout the aneurysm. In certain circumstances, however, the configuration of the TAA allows for partial resection or reconstruction that prepares for further proximal or distal aortic replacement. This staged approach may provide less of a physiological insult and lower risk of paraplegia in these patients. Our previous clinical experience suggests that completion or segmental resection of extensive TAAs months to years after the first aortic replacement results in excellent neurologic outcomes [2]. We present an intentional staged approach in two consecutive cases of extensive TAAs with favorable anatomical configuration.

\footnotetext{
* Corresponding Author:
}

Gabriele Di Luozzo, MD

Hackensack University Medical Center

Cardiothoracic Surgery, 20 Prospect Avenue,

Suite 406, Hackensack, New Jersey 07601, United States

Phone: +1 551996 4785, Fax: +1 551996 4833, E-Mail: gdiluozzo@HackensackUMC.org 


\section{Case Presentation}

\section{Patient 1}

A 78-year-old female with a history of hypertension presented with back pain and was discovered on computerized tomography (CT) scan to have an aortic arch and Type II thoracoabdominal aortic aneurysm (TAA) (Figure 1A). The maximum diameter of the TAA was $8.6 \mathrm{~cm}$ and the aortic arch measured $5.3 \mathrm{~cm}$. In light of the aortic arch aneurysm and extensive distal aortic aneurysmal disease, an aortic arch replacement with elephant trunk was performed. Her postoperative course was uneventful. Due to the high risk (10\%) of spinal cord ischemia in Type II TAA repairs, we decided to approach her aneurysm in a staged manner. The distal portion of the descending thoracic aorta narrowed to $3.5 \mathrm{~cm}$ for a length of 2.5 centimeters before it expanded to a diameter above 6 centimeters in the abdominal aorta segment. We decided to resect the aorta from the narrowed portion to the aortic bifurcation (Type III TAA repair) (Figure 1B). She underwent the extensive aortic resection with hypothermic circulatory arrest $\left(18^{\circ} \mathrm{C}\right)$, sequential ligation of SAs (T10-L5), spinal fluid drainage, and visceral reimplantation with a multi-branched graft (Figure 1B). Somatosensory and motor evoked potentials were performed intraoperatively. Her postoperative course was uneventful. She returned 2 months later for a thoracic endovascular aortic repair (TEVAR) to cover the descending thoracic aortic portion adjoining the elephant trunk to the proximal end of the graft (Figure 1C). The TEVAR was performed under general anesthesia with spinal cord fluid drainage and topical cooling to a bladder temperature of $33^{\circ} \mathrm{C}$. The patient had no clinical evidence of spinal cord injury after each intervention.

\section{Patient 2}

A 67-year-old male with a history of hypertension, hyperlipidemia, and Type B aortic dissection presented with periodic episodes of back pain, early satiety, and a 60 pound weight loss. His CT scan revealed a TAA that spanned from the distal aortic arch to the level of the renal arteries (Figure 2A). The diameter of the segment of aorta at the level of diaphragm was $7 \mathrm{~cm}$, which was displacing and narrowing the esophagogastric junction, resulting in the patient only being able to tolerate a pureed diet. The patient was discharged home for 7 days while his clopidogrel was discontinued and prepared for an elective aortic arch replacement with an elephant trunk. He was readmitted with aspiration pneumonia the day prior to the scheduled operation. A gastrostomy tube was placed for nutritional support. Due to his prolonged pre-operative management, weight loss, and further rapid expansion of his TAA $(9 \mathrm{~cm})$, we decided to change our approach to an open TAA repair and TEVAR of the distal aortic arch and proximal descending thoracic aorta. The CT scan indicated a narrowing of the TAA at the level of the middle of the

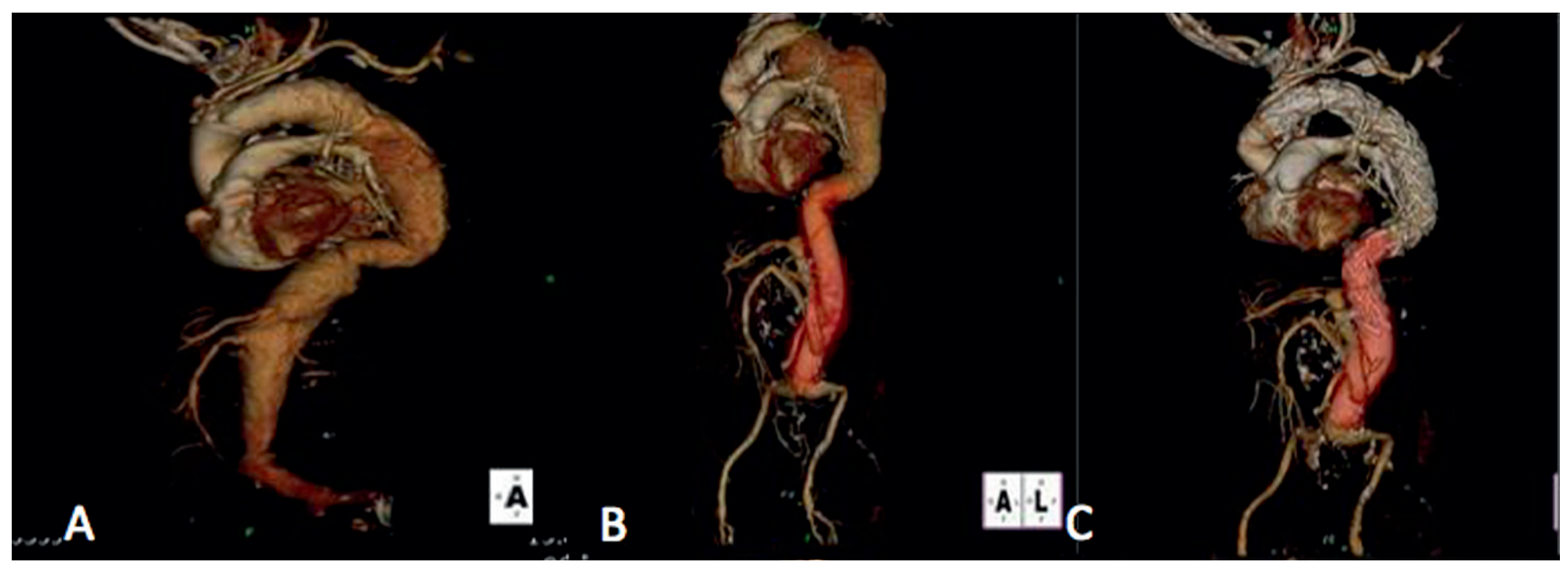

Figure 1. Panel A. Patient 1 pre-operative scan, revealing a distal aortic arch aneurysm and Type II TAA. Panel B. Postoperative CT scan after first stage, showing aortic arch replacement with elephant trunk and Type IV repair (highlighted in red). Panel C. CT scan after second stage with TEVAR. 
descending thoracic aorta (Figure 2A). He underwent a TAA repair from the mid-descending thoracic aorta to below the renal arteries with full cardiopulmonary support $\left(22^{\circ} \mathrm{C}\right)$, spinal fluid drainage, somatosensoryevoked potential (SSEP) and motor-evoked potential (MEP) monitoring, sequential SA ligation (T8-L3), and patch technique to reimplant the visceral vessels (Figure 2B). Six weeks later, he underwent a carotidsubclavian artery bypass and a TEVAR from the left carotid artery to the aortic graft (Figure $2 \mathrm{C}$ ). The patient had no clinical evidence of spinal cord injury.

\section{Discussion}

Paraplegia is a one of the most dreadful complications after TAA repair, carrying a cumulative in-hospital and 1 -year mortality of nearly $80 \%$. Over the last two decades, several adjuncts such as hypothermia, permissive hypertension, and cerebral spinal fluid drainage have been consistently used in clinical practice to prevent $\mathrm{SCl}$. Several centers feel strongly that re-implantation of one or a series of intercostal arteries onto the aortic graft has a positive impact on preventing spinal cord ischemia. Conversely, other surgeons have shown similar neurologic outcomes without implantation of intercostal arteries [1]. We do not feel intercostal artery implantation should be considered unless there neurologic monitoring indicates ischemia, which does not ameliorate with increasing the blood pressure and spinal fluid drainage. The occlusion of greater than 8 SAs and crossing beyond the diaphragm in one setting increases the incidence of $\mathrm{SCl}$. In both cases, the number of SAs involved would have exceeded 12 and with the aneurysms spanning into the abdominal segment, the predicted risk of $\mathrm{SCl}$ could have been as high as $12.5 \%$ [1]. On the contrary, the staging of the TAA repair only required eight SAs to be sacrificed during the open procedure lowering the risk of SCl [1].

Staged repair of thoracoabdominal aortic aneurysms lowers the incidence of paraplegia in experimental animals. The reduction in the CNPP is significantly lower if the sacrifice of SAs is performed in two settings. Our experimental data indicates that the CNPP drops to $80 \%$ of the baseline aortic pressure and requires over 120 hours to return to baseline if all SAs are sacrificed at one setting. These findings were consistent if the SAs were ligated in open repair setting or covered utilizing an endovascular technique. However, when a staged repair was undertaken, the incidence of clinically evident $\mathrm{SCl}$ and histopathological evidence of neuronal injury was decreased significantly [3]. The restoration of the CNPP at 120 hours after SA ligation suggests that the second stage should not performed until after the seventh postoperative days. Interestingly, the order in which the thoracoabdominal aorta was managed; either thoracic first and abdominal aorta to follow or vice versa, the incidence

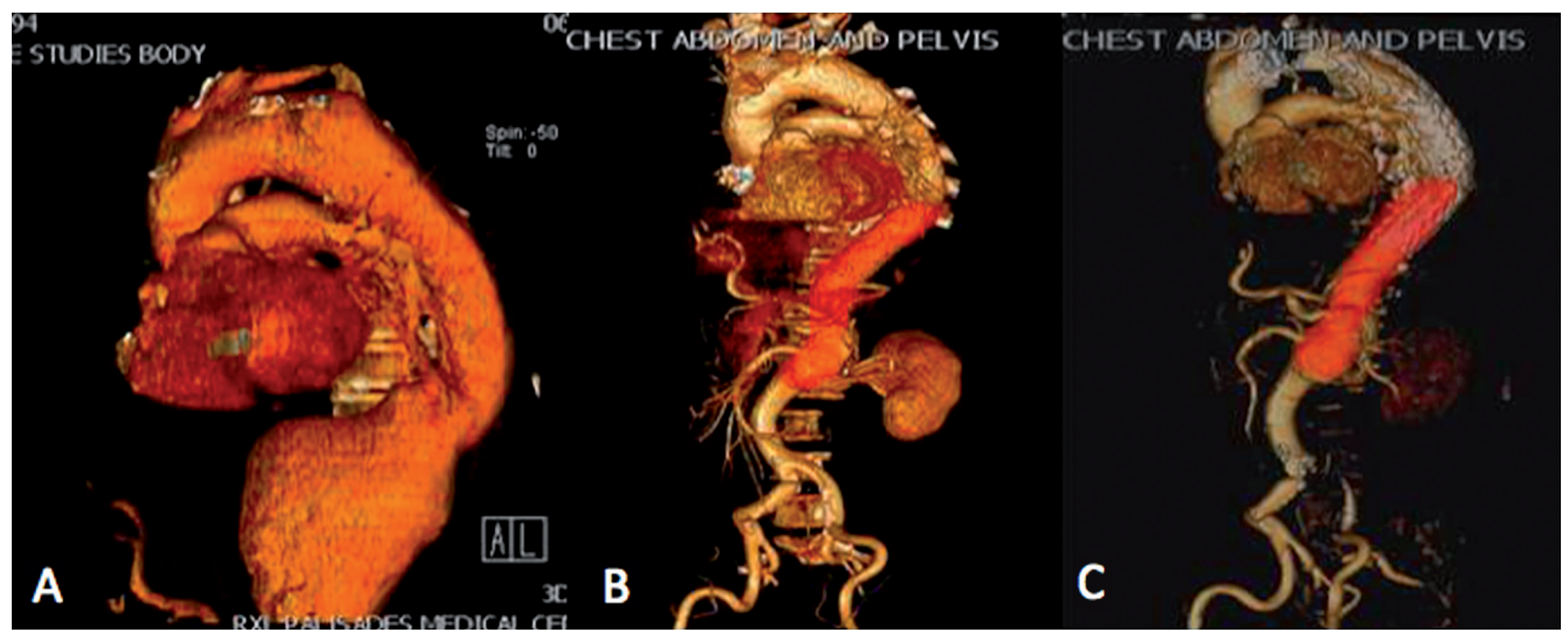

Figure 2. Panel A. Patient 2 with Type III TAA and (Panel B) CT scan following open repair from mid-descending thoracic aorta to below renal arteries (highlighted in red). Panel C. TEVAR repair of distal aortic arch to mid-descending thoracic aorta. 
of $\mathrm{SCl}$ was similar. In both cases, we performed the abdominal aortic portion first; however, this decision was based purely on their particular anatomy. Despite the lower incidence of $\mathrm{SCl}$ we continue to utilize spinal fluid drainage since in the experimental model there was evidence of neuronal damage despite normal motor function.

In conclusion, we present two cases of complex TAA who were approached in a two-stage manner with good clinical outcomes. This approach should be considered when anatomical characteristics are favorable and an extensive aortic replacement is needed. Since this approach requires a lag time between interventions, the risk of waiting needs to be weighed against the risk of rupture of the remaining, untreated aortic aneurysm. Our limited clinical experience with this approach has resulted in acceptable outcomes and an enthusiasm to apply this approach to similar patients.

\section{Conflict of Interest}

The authors have no conflicts of interest relevant to this publication.

\section{Comment on this Article or Ask a Question}

\section{References}

1. Zoli S, Roder F, Etz CD, Brenner RM, Bodian CA, Lin HM, et al. Predicting the risk of paraplegia after thoracic and thoracoabdominal aneurysm repair. Ann Thorac Surg. 2010;90:1237-1244. DOI: 10.1016/j. athoracsur.2010.04.091

2. Etz CD, Zoli $S$, Kari FA, Mueller CS, Bodian CA, Di Luozzo G, et al. Redo lateral thoracotomy for reoperative descending and thoracoabdominal aortic re- pair: A consecutive series of 60 patients. Ann Thorac Surg. 2009;88:756-766. DOI: 10.1016/.athoracsur.2009.04.140

3. Bischoff MS, Scheumann J, Brenner RM, Ladage D, Bodian CA, Kleinman G, et al. Staged approach prevents spinal cord injury in hybrid surgical-endovascular thoracoabdominal aortic aneurysm repair: An experimental model. Ann Thorac Surg. 2011;92:138-146. DOI: 10.1016/j.athoracsur.2011.03.098
Cite this article as: $\mathrm{Di}$ Luozzo G, Wilderman M, Pawale A, McCullough J, Griepp RB. Planned Staged Repair of Thoracoabdominal Aortic Aneurysms to Minimize Spinal Cord Injury. AORTA (Stamford). 2015;3(5):177-180.DOI:http:// dx.doi.org/10.12945/j.aorta.2015.15.003 